\title{
Tapping mode atomic force microscopy in liquid
}

\author{
Constant A. J. Putman, Kees O. Van der Werf, Bart G. De Grooth, Niek F. Van Hulst, \\ and Jan Greve \\ University of Twente, Department of Applied Physics, P.O. Box 217, 7500 AE Enschede, The Netherlands
}

(Received 4 Jantury 1994; accepted for publication 11 March 1994)

\begin{abstract}
We show that standard silicon nitride cantilevers can be used for tapping mode atomic force microscopy (AFM) in air, provided that the energy of the oscillating cantilever is sufficiently high to overcome the adhesion of the water layer. The same cantilevers are successfully used for tapping mode AFM in liquid. Acoustic modes in the liquid excite the cantilever. On soft samples, e.g., biological material, this tapping mode AFM is much more gentle than the regular contact mode AFM. Not only is the destructive influence of the lateral forces minimized, but more important, the intrinsic viscoelastic properties of the sample itself are effectively used to "harden" the soft sample.
\end{abstract}

Recently, a new imaging mode in air, a hybrid form of contact and noncontact mode atomic force microscopy (AFM), was introduced. ${ }^{1,2}$ In this so-called tapping mode, the cantilever (driven by a piezoelectric actuator) vibrates at its resonance frequency. Upon approaching the sample, the tip briefly touches, or taps, the surface at the bottom of each swing, resulting in a decrease in oscillation amplitude. The feedback loop keeps this decrease at a preset value and a topographic image of the sample surface can be obtained. The restoring cantilever force should be higher than the adhesion force due the water film present on samples under ambient conditions. ${ }^{3}$ For this reasun stiff cantilevers ${ }^{4}$ with force constants ranging from 10 to $100 \mathrm{~N} / \mathrm{m}$ and oscillation amplitudes between 50 and $100 \mathrm{~nm}$ are used. In tapping mode AFM, the destructive influence of the lateral forces, due to the relative movement of the tip with respect to the sample, is virtually eliminated, as is the case in noncontact mode AFM, because the duration of tip-sample contact is short. The lateral resolution, however, is as high as in contact mode AFM, being determined by the tip sharpness. A soft viscoelastic polymer can now be imaged without discernible damage.

The disruption of soft samples, e.g., biological samples, reduces significantly when imaging under liquid and removing the high adhesion forces due to the water film on samples under ambient conditions. ${ }^{3}$ However, the lateral forces are still there and tend to wipe away and smear out surface features on soft samples, such as living cells. The operation of tapping mode AFM under liquid could potentially reduce the disruptive intluence of the lateral forces and give less deformation on soft samples.

In this letter we show that tapping mode AFM can be operated in air using standard V-shaped silicon nitride cantilevers $^{2}$ with force constants of 0.38 and $0.58 \mathrm{~N} / \mathrm{m}$. Moreover, with those same cantilevers we have performed tapping mode AFM successfully in liquid.

An existing stand-along AFM in which the tip is scanned, ${ }^{5,6}$ has been modified by positioning a piezo actuator (AE0203D08, NEC, Japan) in the cantilever holder. Raising the driving frequency from 10 to $100 \mathrm{kHz}$ gives the frequency characteristics in air as shown in Fig. 1(a) (dashed line). Many resonances can be observed including the cantilever resonance at $52 \mathrm{kHz}$ with a $Q$ factor of about 40 . Driv- ing the cantilever at $52 \mathrm{kHz}$, while nearing a glass surface, gives an approach curve as shown in Fig. 1(b). Up till point $A$, the cantilever oscillates free from the surface with an amplitude of $450 \mathrm{~nm}$. Beyond $A$, the tip starts touching the surface, causing a shift of the resonance frequency and a decrease in amplitude. Between $A$ and $B$, the amplitude decreases linearly and a feedback loop can be activated, enabling topographic imaging of soft surfaces. From $B$ on, the tip is continuously in contact with the glass surface. ${ }^{7}$ At that point there is not enough energy stored in the cantilever oscillation to overcome the adhesion force exerted on the tip by the water layer [90 $\mathrm{nN}$ in Fig. 1(b)].

The V-shaped silicon nitride cantilevers used for tapping mode AFM in air have force constants $k$ of 0.38 and 0.58 $\mathrm{N} / \mathrm{m}$ and are $100 \mu \mathrm{m}$ long. The $200 \mu \mathrm{m}$ cantilevers, most frequently used in contact mode AFM, are too weak (0.06 and $0.12 \mathrm{~N} / \mathrm{m}$ ) to obtain stable imaging in tapping mode AFM in our setup. There is no fundamental limitation, however, to use these cantilevers in tapping mode AFM in air. In that case, oscillation amplitudes of several micrometers are required. Typically, the oscillation amplitudes are 10 times larger than the amplitudes used in tapping mode AFM with the stiffer silicon cantilevers. ${ }^{1}$ In light of the energy stored in the cantilever movement, $\frac{1}{2} k a^{2}$ (with $a$ the oscillation amplitude), it is clear that $a$ should be 10 times greater if $k$ is roughly 100 times smaller.

Immersing the cantilever in liquid (also the piezo actuator is partially immersed) and raising the driving voltage again from 10 to $100 \mathrm{kHz}$, results in the frequency characteristics as shown in Fig. 1(a) (solid line). The cantilever resonance in air at $52 \mathrm{kHz}$ is damped, but the overall signal level has increased when compared to the situation of air (dashed line). More important, new resonances appear, especially in the 10 to $20 \mathrm{kHz}$ range. Changing the layout of the liquid cell-e.g., larger diameter-or changing the liquid level significantly, results in a shift of the positions of the resonances and a change in their magnitude. The same holds for changing from water to alcohol. These observations point into the direction of the presence of acoustic modes in the medium and the acoustic excitation of the cantilever.

Driving the cantilever at a resonance of $14.1 \mathrm{kHz}$, while approaching a glass surface, gives the curve of Fig. 2(a). Starting with a free oscillation of $30 \mathrm{~nm}$, the cantilever starts 

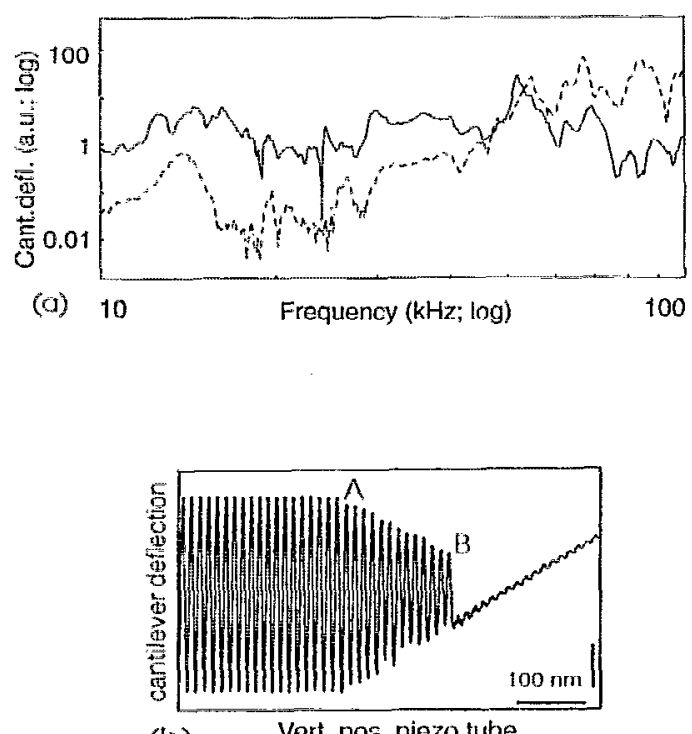

(b)

FIG. 1. (a) Cantilever deflection as a function of the driving frequency $(10-100 \mathrm{kHz})$ in air (dashed line) and liquid (solid line) for a $100 \mu \mathrm{m}$ $V$ shaped silicon nitride cantilever $(k=0.58 \mathrm{~N} / \mathrm{m})$. (b) Tip-sample (glass slide) approach curve in air while oscillating the cantilever at $52 \mathrm{kHz}$. It shows the cantilever deffection as function of the vertical position of the piezotube used for scanning (a voltage ramp is supplied by the $z$ electrode). $A$ and $B ;$ see text.

tapping the surface at point $A$. From $A$ to $B$ the oscillation amplitude decreases, and a feedback loop can be operated. Comparing this approach curve with Fig. 1(b), shows that in air the amplitude decreases symmetrically in the tapping region $(A$ to $B)$, whereas under liquid only the bottom envelope of the oscillation changes. This indicates that in air the cantilever oscillates at its fundamental harmonic resonance frequency. In liquid on the other hand, the AFM is basically operated in a mode taking force-vs-distance curves ${ }^{3}$ at a high rate. From point $B$ on the tip is permanently in contact with the glass surface. ${ }^{7}$ The amplitude between $A$ and $B$ does not decrease linearly as expected, but a contribution from the angular deflection during the period of contact builds up on the decreased amplitude of free oscillation. This is more clearly visualized in Fig. 2(b), where arrows indicate the transitions from free cantilever movement to the pivoting movement during contact. Selecting another resonance from Fig. 1(a) (at $23.6 \mathrm{kHz}$ ) can result in an approach curve as shown in Fig. 2(c). Since there is no region of amplitude decrease before there is an upward shift of the top envelope of oscillation, this resonance mode cannot be used for tapping.

In the region between $A$ and $B$ [Fig. 2(a)] a setpoint can be selected to operate the feedback loop. Since there is no continuous contact between tip and sample as in regular contact AFM, maximum and average loading forces ( $F$ max resp. $F_{\text {av }}$ ) during imaging have to be determined. On the basis of Fig. 2(b) and the assumption that the amplitude of free cantilever movement decreases linearly from $A$ to $B$, we calculate $F_{\max }$ and $F_{\text {av }}$ in point $C$. The rest amplitude of free cantilever movement at $C$ is about $20 \mathrm{~nm}$. Starting out with an amplitude of $30 \mathrm{~nm}$, this implies that the cantilever is deflected $10 \mathrm{~nm}(\Delta x)$ yielding an $F_{\max }$ equal to $3.8 \mathrm{nN}(k$ is $0.38 \mathrm{~N} / \mathrm{m})$. The free movement of the cantilever can be described by a harmonic oscillation with period $T$ and amplitude $A_{0}$. During the period $\tau$ the tip is in contact with the sample. It can be derived that during $\tau$

$$
F_{\mathrm{av}}=k A_{0}\{(T / 2 \pi \tau) \sin (2 \pi \tau / T)-\cos (2 \pi \tau / T)\} .
$$

In approximation this becomes

$$
F_{\mathrm{av}}=k A_{0} \frac{4 \pi^{2}}{3}\left(\frac{\tau}{T}\right)^{2}
$$

with $\tau / T=\arccos \left[\left(A_{0}-\Delta x\right) / A_{0}\right] / 2 \pi$. With $A_{0}=15 \mathrm{~nm}, \Delta x$ $=10 \mathrm{~nm}$ and $k=0.38 \mathrm{~N} / \mathrm{m}$, this results in an average loading force $F_{\text {av }}$ of $2.8 \mathrm{nN}$ at point $C$. This force can be compared with the loading forces in contact mode AFM if the duty cycle $(\tau / T$; equals 0.2 in point $C$ ) is taken into account. The effective average loading force is then $0.55 \mathrm{nN}$.

In Fig. 3(a) a tapping mode AFM image of part of a living monkey kidney cell is shown. The image shows nice details on the cellular surface. The lateral resolution is currently a few tens of nanometers (data not shown). Changing the damping, i.e., shifting setpoint $C$ from $A$ to $B$ [Fig. 2(a)], results in a significant decrease in imaged height of a cell, and a visualization of subsurface features, such as cytoskeletal fibers. ${ }^{8}$ In Fig. 3(b) this is illustrated by line scans on a

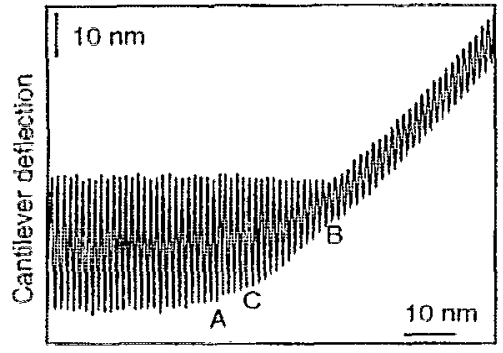

(a)

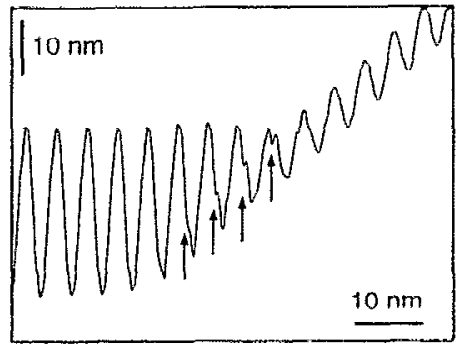

(b) Vertical position piezo tube

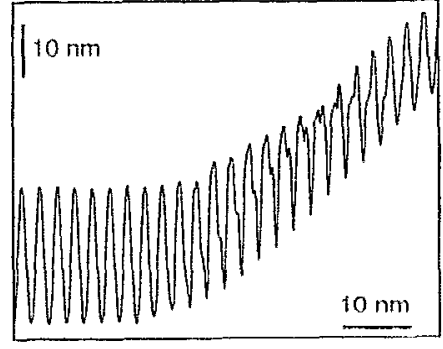

(c)

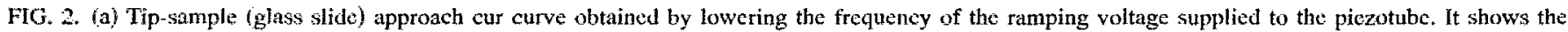

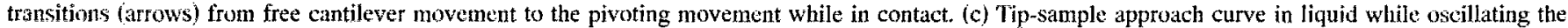

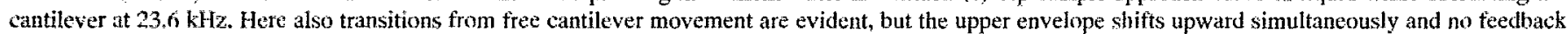
loop (based on amplitude detection) can be operated. 

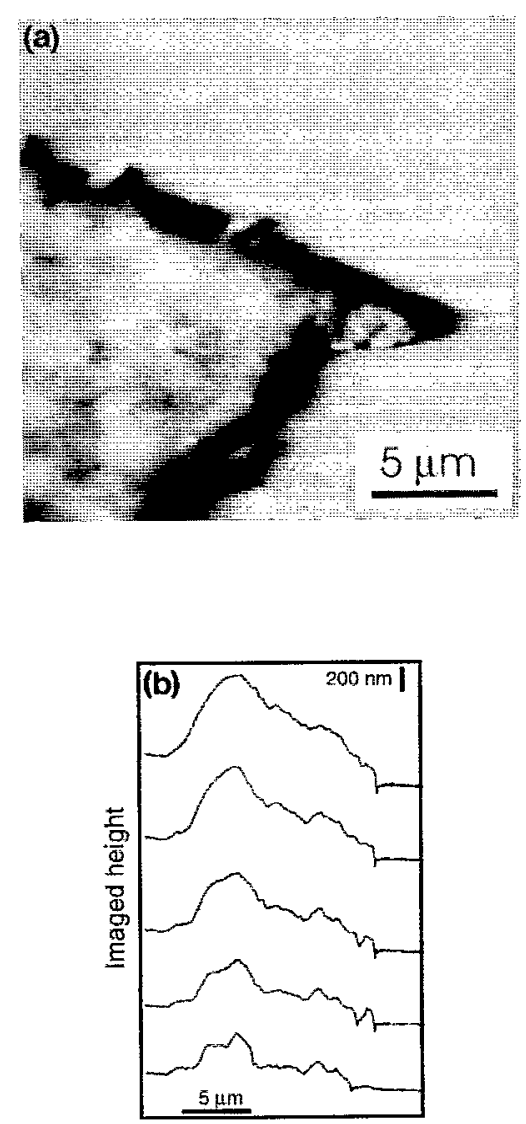

FIG. 3. (a) Tapping mode AFM image of a living monkey kidney cell on glass in growth medium. The tapping amplitude was $25 \mathrm{~nm}$, the resonance frequency was $14.1 \mathrm{kHz}$ and the average loading force during contact was about $2 \mathrm{nN}$. The data has been high-pass filtered to enhance surface features. Image area: $20 \times 20 \mu \mathrm{m}^{2}$. (b) Imaged cell height upon increasing the damping (upper curve: low damping; lower curve: high damping), i.e., shitting the setpoint from $A$ to $B$ [see Fig. 2(a)].

cell monitored under an increasing damping. Recently we have shown that this behavior can be explained by the viscoelastic properties of biological materials. ${ }^{8}$ Under a tapping motion at high frequencies the soft biological materials, such as cell surfaces, behave as "hard" materials. As a direct consequence it becomes less susceptible to deformation. This may be the major reason for the success of the tapping mode AFM and seems as important as the reduction of influence of the lateral forces. Although the lateral forces in tapping mode AFM are comparable to those in contact mode AFM, the energy dissipation by the lateral forces (causing sample degradation) in tapping mode AFM is much smaller. In our case the lateral movement during the period of contact is less than $1 \mathrm{~nm}$ (scan range: $20 \mu \mathrm{m} ; 500$ pixels; 10 cycles per pixel; duty cycle: 0.2 ). It is hard to imagine that any damage can be done by the lateral forces during this brief contact.

In contact mode AFM, the loading force is continuous and the sample relaxates under this load. Although in contact mode AFM the loading force may be lower than in tapping mode AFM the deformation will be higher. In tapping mode AFM one can mimic this situation by taking a setpoint close to $B$ [lower scan line in Fig. 3(b)]. In that case the tip is in contact with the sample most of the time.

Experiments showed that the $200-\mu \mathrm{m}$-long cantilevers do not meet the feedback requirements discussed above. This could possibly be due to the physical characteristics of the cantilever, which do not match the acoustic excitation modes present in our liquid cell. So routinely the $100-\mu \mathrm{m}$-long cantilevers are currently being used with oscillation amplitudes of 200 to $1000 \mathrm{~nm}$ in air and $20-200 \mathrm{~nm}$ in liquid. Most of the resonances in Fig. 1(a) (solid line) cannot be used in tapping mode AFM, but there are always a few resonances in the 10 to $20 \mathrm{kHz}$ range which do meet the feedback requirements. Although the stiff single crystal silicon cantilevers (117 $\mu \mathrm{m}$ long) ${ }^{4}$ can also be used in tapping mode AFM in liquid, their high force constants give high loading forces and tend to damage biological materials; the increase in sample stiffuess (at approximately $200 \mathrm{kHz}$ ) cannot compensate the increase in cantilever stiffness. Further experiments are needed to evaluate the potential application of silicon cantilevers with lower force constants (comparable to those of the cantilevers used in this study) in tapping mode AFM on soft samples in liquid. Higher lateral resolution will probably be obtained, because the tips are sharper than the pyramidal tips on the silicon nitride cantilevers ( $10 \mathrm{~nm}$ vs $20-50$ $\mathrm{nm})$. Supertips on top of the pyramidal tips ${ }^{9}$ can also be used to increase the resolution.

This work was supported by the Dutch Organization for Scientific Research NWO.

${ }^{\prime}$ O. Zhong, D. Inniss, K. Kjoller, and V. B. Elings, Surf. Sci. Lett. 290 , L.688 (1993).

${ }^{2}$ Digital Instruments, Santa Barbara, CA.

${ }^{3}$ A. L. Weisenhorn, P. K. Hansma, T. E. Albrecht, and C. E. Quate, Appl. Phys. Lett. 54, 2651 (1989).

${ }^{4}$ Nanoprobe, Aidlingen, Germany.

${ }^{5}$ C. A. J. Putman, A. M. Van Leeuwen, B. G. De Grooth, K. Radosevic, K. O. Van der Werf, N. F. Van Iulst, and J. Greve, Bioimaging 1, 61 (1993).

${ }^{\circ}$ K. O. Van der Werf, C. A. J. Putman, B. G. De Grooth, F. B. Segerink, E. H. Schipper, N. F. Van Hulst, and J. Greve, Rev. Sci. Instrum. 64, 2892 (1993).

${ }^{7}$ In our setup the cantilever displacement is detected by optical beam deflection [G. Meyer and N. M. Amer, Appl. Phys. Lett. 53, 2400 (1988)]. This technique detects angular deflections of the cantilever which are translated to vertical tip displacenents. Obviously, there is no vertical tip displacement while in contact, but there still is an angular deffection of the cantilever with the tip as the pivoting point.

${ }^{8}$ C. A. J. Putman, K. O. Van der Wert, B. G. De Grooth, N. F. Van Hulst, and J. Greve (unpublished).

${ }^{9}$ D. Keller and C. C. Chou, Surf. Sci. 268, 333 (1992). 